Article

\title{
Investigating farm fragmentation as a risk factor for bovine tu- berculosis in cattle herds: a matched case-control study from Northern Ireland
}

\author{
Georgina Milne ${ }^{1 *}$, Jordon Graham ${ }^{1}$, John McGrath ${ }^{2}$, Raymond Kirke ${ }^{3}$, Wilma McMaster ${ }^{4}$, Andrew William \\ Byrne $^{5}$
}

1. Agri-food and Biosciences Institute (AFBI), Belfast, BT4 3SD, UK; Georgina.milne@afbini.gov.uk (GM); Jordon.Graham@afbini.gov.uk (JG);

2. Department of Agriculture, Environment and Rural Affairs (Area Based Scheme), Orchard House, Foyle Street, Derry/Londonderry, BT48 6AT; John.McGrath@daera-ni.gov.uk (JMcG)

3. Department of Agriculture, Environment and Rural Affairs (Veterinary Service Animal Health), Ballykelly House, Ballykelly Road, Ballykelly, Limavady, BT49 9HP; Raymond.Kirke@daera-ni.gov.uk (RK)

4. Department of Agriculture, Environment and Rural Affairs (Land Parcel Identification System), Academy House, Ballymena, BT43 6HY, UK; Wilma.McMaster@daera-ni.gov.uk (WMcM)

5. One-Health Unit, Department of Agriculture, Food and the Marine, Agriculture House, Dublin, Ireland; AndrewW.Byrne@agriculture.gov.ie (AWB)

* Correspondence: georgina.milne@afbini.gov.uk

\begin{abstract}
Bovine tuberculosis (bTB) remains a challenging endemic pathogen of cattle in many parts of the globe. Spatial clustering of Mycoacterium bovis molecular types in cattle suggests that local factors are the primary drivers of spread. Northern Ireland's agricultural landscape is comprised of highly fragmented farms, distributed across spatially discontinuous land parcels, and these highly fragmented farming structures are thought to facilitate localised spread. We conducted a matched case control study to quantify the risks of bTB breakdown with farm area, farm fragmentation, fragment dispersal, and contact with neighbouring herds. Whilst our results show small but significant increases in breakdown risk associated with each of farm fragmentation, farm area, fragment dispersal, and contact with neighbouring herds, these relationships were strongly confounded with the number of contiguous neighbours with bTB. Our key finding was that every infected neighbour led to an increase in the odds of breakdown by $40 \%$ to $50 \%$, and that highly fragmented farms were almost twice as likely to have a bTB positive neighbour compared to nonfragmented farms. Our results suggest that after controlling for herd size, herd type, spatial and temporal factors, farm fragmentation increasingly exposes herds to infection originating from first order spatial neighbours. Given NI's particularly fragmented landscape, and reliance on shortterm leases, our data supports the hypothesis that between-herd contiguous spread is a particularly important component of NI's bTB disease system.
\end{abstract}

Keywords: Farm fragmentation; bTB; bovine tuberculosis; Northern Ireland; local spread; neighbourhood; matched case-control, conacre.

\section{Introduction}

Bovine tuberculosis (bTB), caused primarily by Mycobacterium bovis infection, is a complex and challenging disease of cattle, endemic in many countries across the globe [1]. The disease continues to blight the cattle industry in the United Kingdom (UK) and Republic of Ireland (ROI), despite long-running test-and-slaughter eradication pro- 
grammes [2-4], ancillary testing [5-7], and surveillance at the abattoir for lesions indicative of bTB $[8,9]$. Failure to eradicate the disease is in part a consequence of multiple non-mutually exclusive infection pathways, which can considerable complexity to disease control [10].

Whilst $M$. bovis can be introduced into a herd via processes operating over relatively long distances (for example, the purchasing of cattle from other herds or marts [11-13]), the persistence and spread of M. bovis in the UK and ROI is understood to be particularly dependent on "local" factors [14-17] These include (but are not limited to) spillback facilitated by direct or indirect contact with infected wildlife [18-21], direct contact with neighbouring herds [16, 22, 23], and a contaminated environment [24].

Farm fragmentation, whereby farms are distributed across multiple, spatially discontinuous land parcels, is particularly common on the island of Ireland [25, 26]. In Northern Irish cattle farms, 35\% of businesses were comprised of five or more fragments [27], comparable to the ROI where $32 \%$ of sampled farms were comprised of five or more fragments [28]. In contrast, a study from GB reported that only $7 \%$ of sampled farms had five or more constituent fragments [29]. Farm fragmentation may contribute to the bTB epidemic by providing more opportunities for direct nose to nose contact between cattle from other farms "over the fence" [16, 22, 23].

Contiguous cattle-to-cattle spread can be counteracted with robust biosecurity measures such as double fencing between parcels with a $3 \mathrm{~m}$ gap between neighbours, and fencing off badger latrines [30]. There is no guarantee, however, that these measures are enacted. In a study of farms in north-west England, farming units had contact with between one and seventeen neighbouring farms [31]. In three separate NIbased studies, 79\% [32] and 67\% [12] of fields permitted contact with neighbouring farms, and cattle were found to spend up to $40 \%$ of their grazing days beside neighbours [33]. To try and control contiguous spread, surveillance in NI involves lateral check tests. These are bTB tests applied to neighbouring herds which are grazed in proximity to a breakdown herd, which are deemed at risk by veterinary inspectors. Lateral check tests involve intensive mapping exercises and biosecurity assessments, but due to the fragmented nature of cattle farms in NI, the process of identifying at-risk herds and parcels can be challenging.

Despite earlier studies alluding to the potential for fragmented farms to disseminate infection $[10,34]$ this exposure's contribution to the epidemic in NI remains poorly understood. Furthermore, to the best of the authors' knowledge, this is the first published research to explicitly explore the risk between farm fragmentation and risk of bTB breakdown within cattle herds. The primary objective of this research, therefore, is to quantify the impact of each of 1) farm fragmentation, 2) fragment dispersal and 3) contiguous contact and 4) farm area, on the odds of bTB breakdown. The secondary objective is to assess for confounding and interaction effects with the presence of neighbours with bTB.

\section{Results}

\subsection{Descriptive results}

The final dataset consisted of 19,008 herds, from which 4,637 (24.4\%) has at least one confirmed bTB breakdown during the three year study period; the spatial distribution of case herds in the whole cattle population is illustrated in Figure 1. These case herds were matched to 4,637 controls who did not have a bTB breakdown between 2015 and 2017, Table 1. The average case farm was 8 ha larger (IQR: 1ha -12ha) than the average control farm, and $41 \%$ of case farms were classified as "Large" (>59.1ha)", compared to $33 \%$ of controls. The average case farm was associated with five additional land parcels than the average control farm, and $21.9 \%$ of case farms were "highly fragmented" 
(8-10 fragments) or "very highly fragmented" (11+ fragments)", compared to $17.7 \%$ of control farms. $30 \%$ of case farms had very highly dispersed fragments $(>3.05 \mathrm{~km}$ between fragment centroids), compared to $26 \%$ of control farms, and $41 \%$ of case farms had the highest levels of contact ( $>4.94 \mathrm{~km}$ of shared boundary) with neighbouring farms, compared to $33 \%$ of control farms. Some $71 \%$ of case farms had at least one contiguous neighbour with a confirmed bTB breakdown (up to a maximum of 14), compared to a $53 \%$ of control farms (up to a maximum of 11 ).

Table 1. The distribution of each explanatory variable in the cases, control sample, and whole cattle population. The data were matched on variables Year, DVO, Herd Type and Median herd size. Counts $(\mathrm{N})$ and percentages $(\%)$ are reported for categorical variables, and medians with IQR's are reported for continuous variables.

\begin{tabular}{|c|c|c|c|}
\hline & \multicolumn{3}{|c|}{ bTB Status } \\
\hline & Neg., $N=4,637$ & Pos., $\mathrm{N}=4,637$ & Pop., $N=57,0241$ \\
\hline \multicolumn{4}{|l|}{ Year } \\
\hline 2015 & $1,774(38 \%)$ & $1,774(38 \%)$ & $19,008(33 \%)$ \\
\hline 2016 & $1,389(30 \%)$ & $1,389(30 \%)$ & $19,008(33 \%)$ \\
\hline 2017 & $1,474(32 \%)$ & $1,474(32 \%)$ & $19,008(33 \%)$ \\
\hline \multicolumn{4}{|l|}{ DVO } \\
\hline Armagh & $428(9.2 \%)$ & $428(9.2 \%)$ & $5,487(9.6 \%)$ \\
\hline Ballymena & $269(5.8 \%)$ & $269(5.8 \%)$ & $3,465(6.1 \%)$ \\
\hline Coleraine & $590(13 \%)$ & $590(13 \%)$ & $6,450(11 \%)$ \\
\hline Dungannon & $590(13 \%)$ & $590(13 \%)$ & $6,954(12 \%)$ \\
\hline Enniskillen & $566(12 \%)$ & $566(12 \%)$ & $7,848(14 \%)$ \\
\hline Larne & $226(4.9 \%)$ & $226(4.9 \%)$ & $3,882(6.8 \%)$ \\
\hline Londonderry & $119(2.6 \%)$ & $119(2.6 \%)$ & $2,061(3.6 \%)$ \\
\hline Newry & $688(15 \%)$ & $688(15 \%)$ & $8,934(16 \%)$ \\
\hline Newtownards & $515(11 \%)$ & $515(11 \%)$ & $4,458(7.8 \%)$ \\
\hline Omagh & $646(14 \%)$ & $646(14 \%)$ & $7,470(13 \%)$ \\
\hline NA & - & - & $15(<0.1 \%)$ \\
\hline \multicolumn{4}{|l|}{ Herd Type } \\
\hline Breeder & $1,251(27 \%)$ & $1,251(27 \%)$ & $28,415(50 \%)$ \\
\hline Dairy & $1,267(27 \%)$ & $1,267(27 \%)$ & $8,128(14 \%)$ \\
\hline Finisher & $690(15 \%)$ & $690(15 \%)$ & $5,976(10 \%)$ \\
\hline Other & $1,429(31 \%)$ & $1,429(31 \%)$ & $14,505(25 \%)$ \\
\hline Median herd size & $81(41,156)$ & $83(40,173)$ & $40(19,89)$ \\
\hline Total farm area (ha) & $42(26,72)$ & $50(27,84)$ & $31(16,59)$ \\
\hline \multicolumn{4}{|l|}{ Farm area category ${ }^{1}$} \\
\hline Small & $583(13 \%)$ & $538(12 \%)$ & $14,164(25 \%)$ \\
\hline Medium & $938(20 \%)$ & $852(18 \%)$ & $14,353(25 \%)$ \\
\hline Large & $1,570(34 \%)$ & $1,343(29 \%)$ & $14,255(25 \%)$ \\
\hline Very Large & $1,546(33 \%)$ & $1,904(41 \%)$ & $14,252(25 \%)$ \\
\hline $\mathrm{N}$ fields & $28(17,44)$ & $33(20,51)$ & $24(14,39)$ \\
\hline $\mathrm{N}$ fragments & $4(2,6)$ & $4(3,7)$ & $3(2,6)$ \\
\hline \multicolumn{4}{|l|}{ Fragmentation category ${ }^{2}$} \\
\hline Not fragmented & $526(11 \%)$ & $488(11 \%)$ & $4,691(8.2 \%)$ \\
\hline Little fragmentation & $2,226(48 \%)$ & $1,974(43 \%)$ & $27,164(48 \%)$ \\
\hline Medium fragmentation & $1,062(23 \%)$ & $1,165(25 \%)$ & $12,353(22 \%)$ \\
\hline High fragmentation & $415(8.9 \%)$ & $551(12 \%)$ & $9,476(17 \%)$ \\
\hline Very high fragmentation & $408(8.8 \%)$ & $459(9.9 \%)$ & $3,340(5.9 \%)$ \\
\hline Median distance fragments $(\mathrm{km})$ & $1.52(0.72,3.23)$ & $1.83(0.86,3.57)$ & $1.38(0.52,3.05)$ \\
\hline
\end{tabular}




\begin{tabular}{cccc} 
Fragment distance category $^{3}$ & & & \\
Low & $798(17 \%)$ & $697(15 \%)$ & $14,255(25 \%)$ \\
Medium & $1,338(29 \%)$ & $1,116(24 \%)$ & $14,256(25 \%)$ \\
High & $1,282(28 \%)$ & $1,425(31 \%)$ & $14,256(25 \%)$ \\
Very High & $1,219(26 \%)$ & $1,399(30 \%)$ & $14,255(25 \%)$ \\
\hline Total shared boundary (km) & $3.4(2.0,5.7)$ & $4.1(2.2,6.7)$ & \\
\hline Neighbour contact category ${ }^{4}$ & & & \\
Low & $763(16 \%)$ & $644(14 \%)$ & $14,256(25 \%)$ \\
Medium & $1,086(23 \%)$ & $918(20 \%)$ & $14,256(25 \%)$ \\
High & $1,255(27 \%)$ & $1,181(25 \%)$ & $14,256(25 \%)$ \\
Very High & $1,533(33 \%)$ & $1,894(41 \%)$ & $14,256(25 \%)$ \\
\hline Count neighbours bTB & $1(0,1)$ & $1(0,3)$ & $0(0,1)$ \\
\hline Any neighbour bTB ${ }^{5}$ & $2,463(53 \%)$ & $3,284(71 \%)$ & $27,074(48 \%)$ \\
\hline
\end{tabular}

\footnotetext{
${ }^{1}$ Total farm area (ha) categorized; ${ }^{2} \mathrm{~N}$ fragments categorized; ${ }^{3}$ Median distance fragments $(\mathrm{km})$ categorised
}

${ }^{4}$ Total shared boundary $(\mathrm{km})$ categorized; ${ }^{5}$ Count neighbours bTB dichotimised

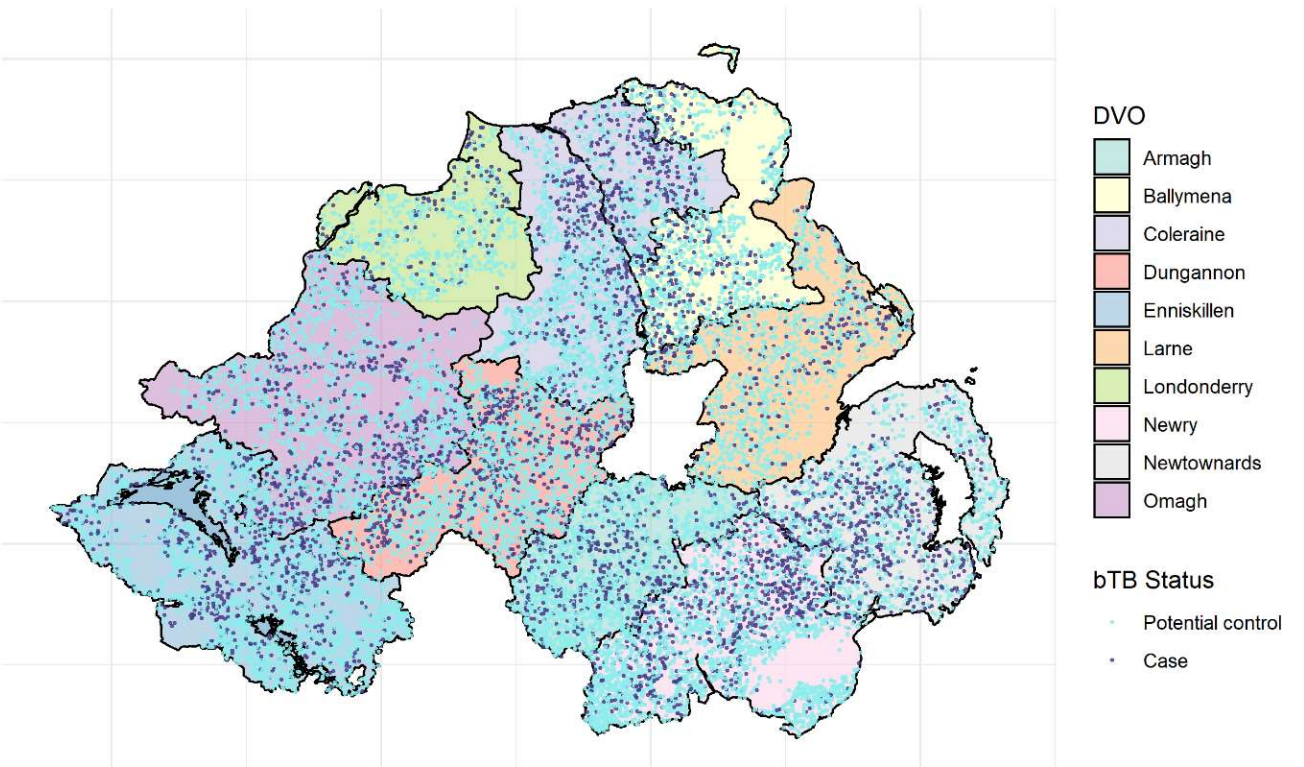

Figure 1. Spatial distribution of case herds and all potential control herds across the $10 \mathrm{DVO}$ areas in NI.

\subsection{Univariable models}

Moderate to strong correlation was observed between farm area, farm fragmentation, fragment dispersal and contact with neighbouring farms (R-Markdown Supplementary Material), with the strongest correlation between the number of fragments and the extent of shared boundary $(\varrho=0.7)$. The continuous distributions of farm area, number of fragments, fragment dispersal, contact category and the number of $\mathrm{bTB}$ positive neighbours are shown in Figure 2A, 2C, 2E, 2G and 2I.

The results of the univariable analyses are shown in Table 2. The LOESS plot of the relationship between bTB status and farm area is shown in Figure 2A. Whilst no association was observed between risk of bTB breakdown and farm area in the continuous 
model, the categorical model shows that only the very largest farms (> 59.1 ha) were associated with elevated bTB positive status, compared to medium sized farms (16.41 ha 31.2 ha, OR: 1.87; 95\% CI: 1.55 - 2.25), see Figure 2B. A positive relationship can also be observed between the number of fragments and bTB positive status in the exploratory LOESS plot (Figure 2C), with every additional fragment linked to small increase in the odds of bTB breakdown (OR: 1.03; 95\%CI: 1.02-1.05). Farm fragmentation was also entered as a categorical variable, and this model shown that in very highly fragmented farms (11+ fragments), the odds of bTB positive status was between $12 \%$ and $65 \%$ more likely than in farms with little fragmentation (2-4 fragments, OR: 1.36; 95\%CI: 1.12-1.65), see Figure 2D. A positive trend between the odds of bTB positive status and fragmentation level was apparent. The test for linear trend reveals that the ordinal model was a poorer fit compared to the categorical model (Chi-Sq $=17.2, \mathrm{df}=3, \mathrm{p}<0.05)$. 

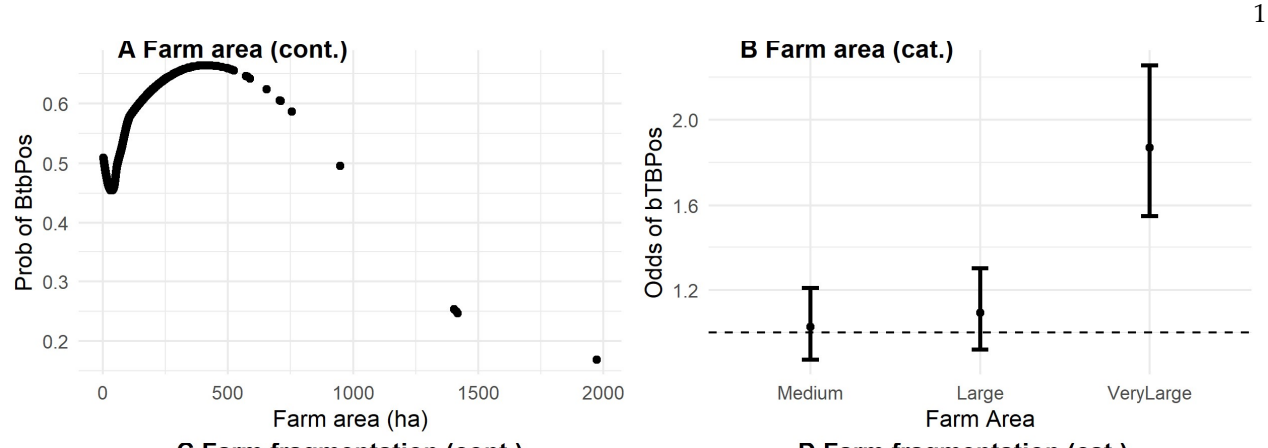

C Farm fragmentation (cont.)
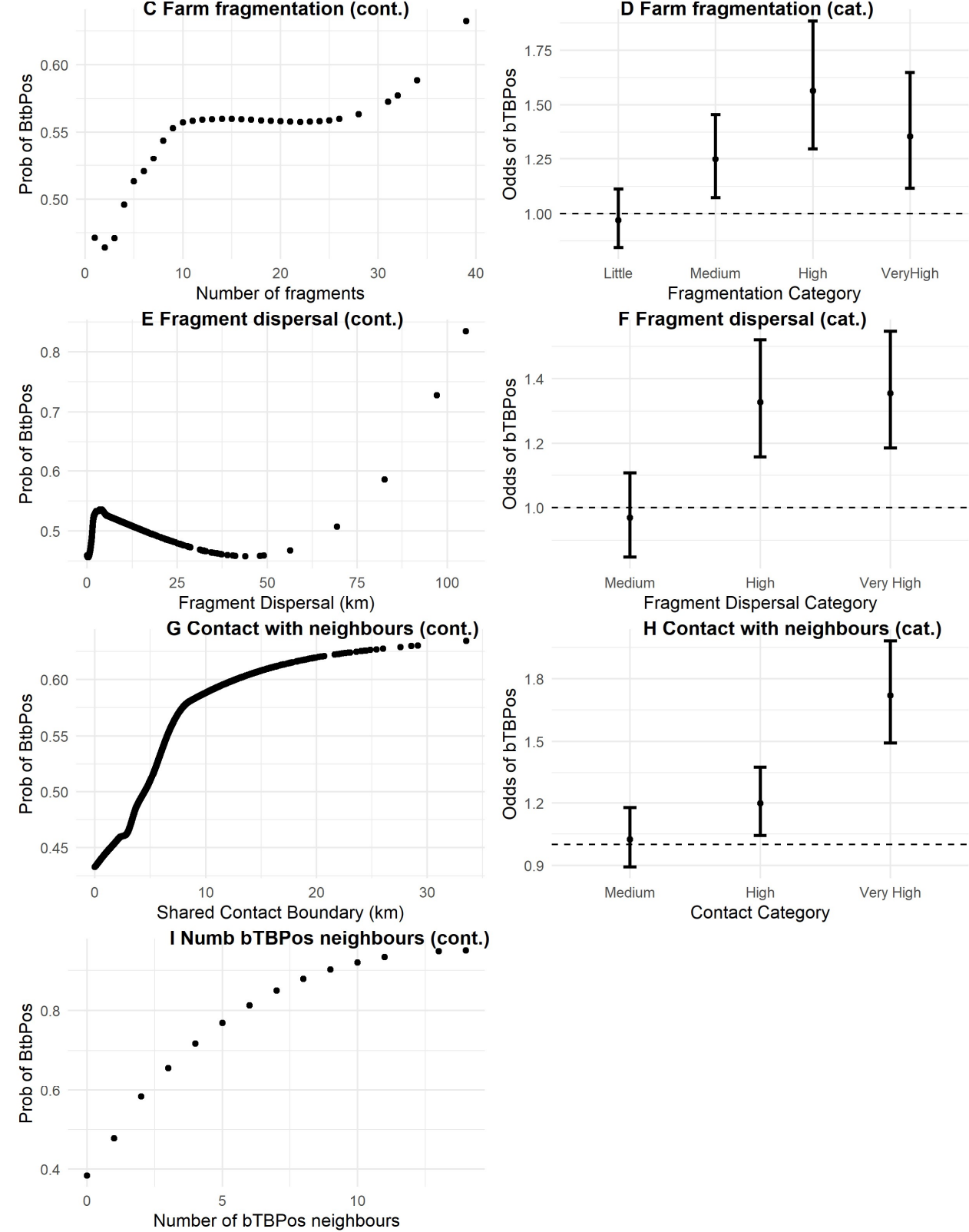

Figure 2. Exploratory LOESS curves (A, C, E, G, I) of continuous predictors plotted against proba- 3 bility of bTB breakdown, and plots of OR's and 95\%CI (B, D, F, H) for each factor in categorical 4 predictors. 
Table 2. Results of the univariable analysis for both categorical and continuous predictors. For continuous predictors, the Odds Ratio (OR) and 95\% lower and upper confidence intervals (95\%CI) are shown. For categorical predictors, the OR and $95 \% \mathrm{CI}$ are also shown, with reference to the baseline category.

\begin{tabular}{|c|c|c|c|c|c|}
\hline & \multicolumn{2}{|c|}{ Categorical } & & \multicolumn{2}{|c|}{ Continuous } \\
\hline Variable & OR & $95 \% \mathrm{CI}$ & & OR & $95 \% \mathrm{CI}$ \\
\hline Farm area category & & & Total farm area (per ha) & 1.00 & $1.00,1.00$ \\
\hline Small (1.2 ha - 16.4 ha $)$ & - & - & & & \\
\hline Medium (16.41 ha - 31.2 ha) & 1.03 & $0.87,1.21$ & & & \\
\hline Large (31.21 ha -59.1 ha) & 1.09 & $0.92,1.30$ & & & \\
\hline Very Large (> 59.1 ha) & 1.87 & $1.55,2.25$ & & & \\
\hline Fragment category & & & $\mathrm{N}$ fragments (per fragment) & 1.03 & $1.02,1.05$ \\
\hline Not_fragmented (1 fragment) & - & - & & & \\
\hline Little fragmentation (2-4 fragments) & 0.97 & $0.84,1.11$ & & & \\
\hline Medium_fragmentation (5-7 fragments) & 1.25 & $1.07,1.46$ & & & \\
\hline High_fragmentation (8-10 fragments) & 1.56 & $1.30,1.88$ & & & \\
\hline $\begin{array}{c}\text { Very_high_fragmentation (11+ frag- } \\
\text { ments) }\end{array}$ & 1.36 & $1.12,1.65$ & & & \\
\hline Fragment distance category & & & $\begin{array}{l}\text { Median distance fragments (per } \\
\qquad 10 \mathrm{~km})^{1}\end{array}$ & 1.00 & $1.00,1.00$ \\
\hline Low $(0 \mathrm{~km}-0.52 \mathrm{~km})$ & - & - & & & \\
\hline Medium $(0.53 \mathrm{~km}-1.38 \mathrm{~km})$ & 0.97 & $0.85,1.11$ & & & \\
\hline High $(1.39 \mathrm{~km}-3.05 \mathrm{~km})$ & 1.33 & $1.16,1.52$ & & & \\
\hline Very High $(>3.06 \mathrm{~km})$ & 1.35 & $1.19,1.55$ & & & \\
\hline Neighbour contact category & & & Total shared boundary (per km) & 1.07 & $1.06,1.09$ \\
\hline Low $(0 \mathrm{~km}-1.48 \mathrm{~km})$ & - & - & & & \\
\hline Medium $(1.49 \mathrm{~km}-2.84 \mathrm{~km})$ & 1.02 & $0.89,1.18$ & & & \\
\hline High $(2.85 \mathrm{~km}-4.95 \mathrm{~km})$ & 1.20 & $1.04,1.38$ & & & \\
\hline Very High $(>4.96 \mathrm{~km})$ & 1.72 & $1.49,1.98$ & & & \\
\hline Any neighbour bTB & 2.20 & $2.01,2.41$ & Count neighbours bTB & 1.45 & $1.40,1.50$ \\
\hline
\end{tabular}

BTB breakdown risk was also associated with fragment dispersal (OR: 1:001; 95\%CI: 1.00-1.002 per 10km), see LOESS plot in Figure 2E, however the effect sizes were very small $(\sim 0.1 \%$ increase in the probability of bTB breakdown per $10 \mathrm{~km}$ between fragments). When modelled as a categorical predictor, bTB breakdowns were between $19 \%$ and 55\% more likely in farms with a "Very High" fragment dispersal (distances $>3.06$ $\mathrm{km}$ between fragments) compared to farms with "Medium" fragment dispersal (distances $0.53 \mathrm{~km}-1.38 \mathrm{~km}$ between fragments; OR: 1.35, 95\%CI: 1.19-1.55); Figure 2F. There was also insufficient evidence that a model of linear trend was a better for compared to a categorical model $(\mathrm{Chi}-\mathrm{Sq}=12.2, \mathrm{df}=2, \mathrm{p}<0.05)$.

The odds of bTB breakdown increased by between $6 \%$ and $9 \%$ for every additional $\mathrm{km}$ of shared boundary with neighbouring cattle farms (OR: 1.07, 95\%CI: 1.06-1.09); see loess plot Figure $3 \mathrm{H}$. The categorical model shows that the odds of bTB breakdown is elevated in farms with "Very High" levels of shared contact boundary (distances $>4.96$ $\mathrm{km}$ ) compared to medium levels (distances $1.49 \mathrm{~km}-2.84 \mathrm{~km}$, OR: 1.72, 95\%CI: 1.49-1.58). The model of linear trend was a poorer fit to these data compared to the categorical model $($ Chi-Sq $=14.7, \mathrm{df}=2, \mathrm{p}<0.05)$.

The number of bTB positive neighbours was also positively associated with the odds of bTB breakdown; for every additional neighbour, the odds increased by between 
40\% and 50\% (OR: 1.45, 95\%CI: 1.40-1.50), see LOESS plot Figure 3I. Likewise, compared to having no neighbours with $\mathrm{bTB}$, having at least one bTB positive neighbour increased the odds of breakdown by between 100 and 141\% (OR: 2.20, 95\%CI: 2.01-2.41).

\subsection{Confounding}

It was not possible to build a model with each of the exposures included, in part because of the extent of correlation between explanatory variables, and also because such a model has very sparsely populated categories. Table 3 shows the crude unadjusted ORs for univariable relationships compared with adjusted ORs (aORs) for each of farm area, farm fragmentation, fragment dispersal and contact with neighbours after controlling for the presence of bTB positive neighbours. In the categorical model for farm area, it was observed that an association between breakdown risk and farm size was apparent in very large farms only, and so to simplify the analysis, a new variable was created dichotomised at this point (>59.1 ha). Given that the OR for this variable was reduced by $22 \%$ once the number of bTB positive neighbours was taken into account, we considered the number of bTB positive neighbours as a positive confounder, making the positive relationship between farm area and bTB status appear stronger than actual. Further investigation of this confounding revealed that the average very large farm had $1 \mathrm{bTB}$ positive neighbour (IQR: 1-3, max =14), compared to the average small farm (0 bTB positive neighbours, IQR 0-3, max =5), see Figure 3A. Furthermore, 75\% of very large farms had at least one bTB positive neighbour, compared to $37 \%$ of smaller farms. However, even after accounting for the number of bTB positive neighbours, very large farms were still positively associated with bTB breakdown risk, compared to smaller farms (aOR: 1.35, 95\%CI: 1.20-1.52).

Confounding effects were also found between farm fragmentation and bTB positive neighbours; in very highly fragmented farms, the OR for bTB positivity was reduced by $46 \%$ once the number of infected neighbours was included in the model; concurrent with this, we observed up to $14 \mathrm{bTB}$ positive neighbours in very highly fragmented farms, compared to a maximum of 5 in non-fragmented farms; Figure 3B. Some $84 \%$ of very highly fragmented farms had at least one bTB positive neighbour, compared to $45 \%$ of non-fragmented farms. After controlling for the presence of infected neighbours, there was no clear association between bTB breakdowns and different levels of farm fragmentation compared to the baseline (medium fragmentation).

The relationship between fragment dispersal and the odds of bTB breakdown was positively confounded with the number of bTB positive neighbours, Figure $3 \mathrm{C}$. Some $42 \%$ of farms with low dispersal have at least one bTB positive neighbour, compared to $68 \%$ of very highly dispersed farms, and farms with very high dispersal were associated with elevated numbers of bTB positive neighbours. After controlling for the presence of infected neighbours, no clear associating remained between the odd of bTB breakdown and fragment dispersal.

Considerable confounding was also identified between neighbour contact category and the presence of bTB positive neighbours. After accounting for the number of infected neighbours, the OR for the category representing high contact between neighbours was reduced by $11 \%$, whilst the OR of the category representing very high contact between neighbours was reduced by $41 \%$. Thirty-six percent of farms with low contact metrics had at least one bTB positive neighbour, compared to $79 \%$ of farms with very high contact, and farms with very high contact metrics also had the largest number of bTB positive neighbours; see Figure 3D. 


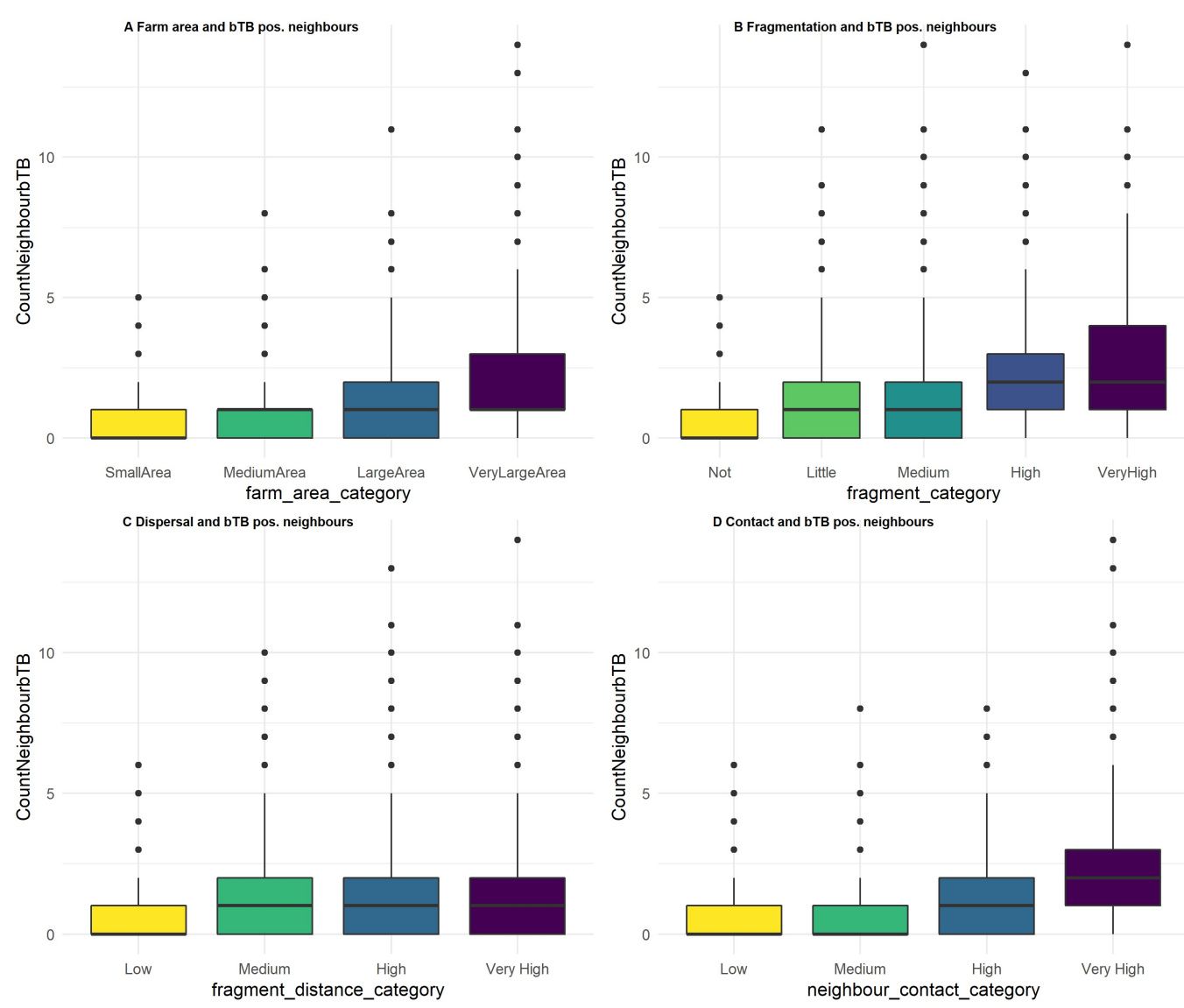

Figure 3. Illustration of confounding between the number of bTB positive neighbours and each of farm area, farm fragmentation, fragment dispersal and contact with neighbouring farms. 
Table 3. The crude ORs and adjusted ORs showing how the number of neighbours with bTB confounds the relationship between each of farm area, farm fragmentation, fragment dispersal and contact with neighbouring farms.

\begin{tabular}{|c|c|c|c|c|c|}
\hline \multirow[t]{2}{*}{ Model } & \multirow[t]{2}{*}{ Variable } & \multicolumn{2}{|c|}{ Unadjusted Analysis } & \multicolumn{2}{|c|}{ Adjusted Analysis } \\
\hline & & OR & $95 \% \mathrm{CI}$ & $\mathrm{aOR}$ & $95 \% \mathrm{CI}$ \\
\hline \multirow{4}{*}{ Farm area } & Farm area category ${ }^{1}$ & & & & \\
\hline & Small/Medium/Large & - & - & - & - \\
\hline & Very Large & 1.74 & $1.56,1.95$ & 1.35 & $1.20,1.52$ \\
\hline & Count neighbours bTB & 1.45 & $1.40,1.50$ & 1.43 & $1.38,1.48$ \\
\hline \multirow{7}{*}{ Fragmentation } & Fragment category & & & & \\
\hline & Not fragmented & - & - & - & - \\
\hline & Little fragmentation & 0.97 & $0.84,1.11$ & 0.83 & $0.72,0.96$ \\
\hline & Medium fragmentation & 1.25 & $1.07,1.46$ & 0.96 & $0.81,1.12$ \\
\hline & High fragmentation & 1.56 & $1.30,1.88$ & 1.06 & $0.87,1.30$ \\
\hline & Very high fragmentation & 1.36 & $1.12,1.65$ & 0.73 & $0.59,0.91$ \\
\hline & Count neighbours bTB & 1.45 & $1.40,1.50$ & 1.45 & $1.40,1.51$ \\
\hline \multirow{6}{*}{ Fragment dispersal } & Fragment distance category & & & & \\
\hline & Low & - & - & - & - \\
\hline & Medium & 0.97 & $0.85,1.11$ & 0.84 & $0.73,0.97$ \\
\hline & High & 1.33 & $1.16,1.52$ & 1.01 & $0.87,1.17$ \\
\hline & Very High & 1.35 & $1.19,1.55$ & 1.01 & $0.88,1.16$ \\
\hline & Count neighbours bTB & 1.45 & $1.40,1.50$ & 1.44 & $1.39,1.49$ \\
\hline \multirow{6}{*}{ Neighbour contact } & Neighbour contact category & & & & \\
\hline & Low & - & - & - & - \\
\hline & Medium & 1.02 & $0.89,1.18$ & 0.91 & $0.79,1.05$ \\
\hline & High & 1.20 & $1.04,1.38$ & 0.95 & $0.82,1.10$ \\
\hline & Very High & 1.72 & $1.49,1.98$ & 1.02 & $0.87,1.19$ \\
\hline & Count neighbours bTB & 1.45 & $1.40,1.50$ & 1.44 & $1.39,1.49$ \\
\hline
\end{tabular}

${ }^{1}$ Dichotimised at "Very large farms"

\section{Discussion}

Previous molecular studies from NI have revealed considerable spatial clustering of $M$. bovis genetic types in host populations, thereby affirming the important role of geographically localised processes in maintaining the bTB epidemic [42-45]. Although NI's highly fragmented farmland is thought to be a contributing factor prolonging the epidemic, no studies to date have explored farm fragmentation as a risk factor for bTB, necessitating a deeper delve into the role of farm fragmentation as a facilitator of localised disease spread. Our principal finding is that after controlling for herd size, herd type, and spatial and temporal factors, increasingly fragmented farms were exposed to greater numbers of first order spatial neighbours with bTB, which was directly associated with increases in bTB breakdown risk.

In our univariable models, we found that farm fragmentation, fragment dispersal and contact with neighbouring farms are each associated with increased odds of bTB breakdown, which is in concordance with previous studies. In the ROI, Byrne et al. (2020) observed an increase in bTB breakdown length of $6.6 \%$ in herds residing on farms 
with four parcels, compared to herds in farms with one parcel [28]. In the randomized badger culling trial (RBCT) area, Johnston et al. (2005) found that operating a farm over multiple premises was linked to a 79\% increase in the odds of a bTB breakdown [46]. A later study in the same area also found that the number of contiguous holdings was an important risk factor for breakdowns [47]. In our study, these three variables of interest (farm fragmentation, fragment dispersal and contact with neighbouring farms) were, however, strongly confounded with the number of first order spatial neighbours with bTB. Once the number of bTB positive neighbours was taken into account, the impact of each of these three factors on bTB breakdown risk was less clear. Our data therefore supports the hypothesis that fragmentation metrics are largely proxy measures of exposure to neighbouring herds, and that the number of contiguous infected herds is therefore the main exposure of interest. Notwithstanding this, fragment dispersal could contribute to spreading disease from higher incidence areas to lower incidence areas via intra-herd movements. Indeed, a consequence of fragmentation is that parcels may be very widely dispersed; $25 \%$ of NI farms had a median distance of $3 \mathrm{~km}$ or more between fragments (to a maximum of $152 \mathrm{~km}$ ) [27]. Unrecorded cattle movements between dispersed, but otherwise connected holdings could drive disease spread and intensify surveillance efforts [48]. However, the strong geographic clustering of $M$. bovis genetic types in NI, along with the relatively small median distances between fragments in the vast majority of farms, means that the importance of long range introductions is not evident. We do acknowledge that moving cattle between fragments could be a potential mechanism of disease spread within the herd, but because the distances involved are small, we argue that disease associated with intra-parcel movements between fragments should be practically indistinguishable from other short-range processes. Simulations of food-and-mouth spread between separate but associated premises in Scotland also suggest a diminished role for long-range intra-herd movements, but highlight the need to better understand land parcel occupancy to fully gauge the impact of intra-premises spread [49].

"Farm area" was the only variable where residual bTB breakdown risk remained after controlling for the presence of bTB positive neighbours; in very large farms $(>59.1$ ha) the odds of breakdown remained elevated by between $20 \%$ and $52 \%$ compared to other farm sizes. This suggests that herds in very large farms experience additional risk beyond that presented by infection from neighbours. It may be that larger farms simply have more badgers on the land, as suggested by Vial et al. [15], or represent farms with mixed grazing of cattle and sheep, which has been linked to prolonged breakdowns in NI [50]. Additionally, the correlation between the variables used in this analysis means that larger farms are also more likely to be more fragmented [27], so the elevated risk linked to larger farms and more fragmented farms may instead represent additional positive confounding between these two exposures.

The number of first-order spatial neighbours with bTB exhibited a particularly strong association with bTB breakdown risk; indeed, the presence of at least one neighbour with bTB was associated with more than double the odds of bTB breakdown compared to having no bTB positive neighbours. In some breakdown herds, up to $14 \mathrm{bTB}$ positive first-order spatial neighbouring herds were identified. The importance of neighbourhood has been highlighted in an ROI study, where $35 \%$ of bTB animal incidence was associated with being within $1 \mathrm{~km}$ of other infected herds [22]. In a Northern Irish case-control study of 427 dairy herds, Denny and Wilesmith (1999) also found that case farms could be over twice as likely to have infected contiguous neighbours [32], and a study of 200 herds in the ROI found that the odds of an animal failing a tuberculin test increased by up for five-fold when a neighbouring farm was restricted in the prior six months [23]. Positive relationships between the odds of bTB breakdown and the number of confirmed breakdowns in neighbouring herds has also been found in UK cattle herds [16]. Whilst the presence of infected neighbours is therefore important in the UK 
and ROI, farm fragmentation is particularly prevalent in NI compared to other areas [29]. We therefore argue that herds in NI may be particularly exposed to disease originating from contiguous neighbours compared to infection from other sources, such as bought-in cattle [51]. Our study did not account for badger density or prevalence, however, and it is likely that wildlife acts as an ongoing infection source within nearby herds, in the absence of any contiguous transmissions. To overcome this limitation, we matched case and control herds to herds within the same geographic area (DVO); arguably case herds and control herds should therefore be exposed to similar risk from local wildlife. We do acknowledge, however, that our approach could overlook within-DVO variation at smaller geographic scales.

Ultimately, tackling lateral spread of infection, requires understanding the drivers of fragmentation itself. Around 30\% of NI's farmland is dominated by the practice of informal short-term land-leasing called "conacre", whereby a landowner may rent out only a portion of their farm for contracts $<12$ months. Leasing conacre is understood to be a pervasive mechanism driving increasing farm fragmentation, and the short-term nature of the lease means that land owners may be less likely to invest in conacre holdings, with subsequent poorer soil, reduced animal productivity and poorer biosecurity $[52,53]$. The conacre system is usually thought of as a factor impacting farm incomes and production, but we argue there may be an important role in conacre as an epidemiological driver of disease, leading to increased exposure between contiguous herds and potentially presenting with poorer biosecurity. Indeed, farmland fragmentation leads to substantial administrative and surveillance activities for DAERA staff to identify neighbours who may be at risk, extending past cattle on directly contiguous fragments at the time of breakdown; candidate neighbours for lateral tests include cattle grazed next to a breakdown herd in previous seasons, or who may graze next to the breakdown herd in future during the course of the breakdown. Consideration is also given to whether break-out cattle may spread infection to neighbouring herds, or whether there is contact with cattle along a laneway as cattle are herded between parcels. Risk of spread via possible wildlife-cattle contact or other indirect means is also considered. All at risk neighbours must be contacted (irrespective of biosecurity between parcels), and short interval full herd tests are carried out where risk of disease spread has been identified. The high levels of fragmentation and dispersal mean that large geographic extents may be involved, requiring local knowledge of the land and the people who farm it.

\subsection{Limitations}

As this was a large scale computation study and not a field study, we were unable to accurately geo-reference herds within holdings. Potentially therefore, herds could occupy only one area within a very fragmented farm. However, a small study quantifying intra-herd cattle movements in NI shows that even fragmented land was grazed frequently, especially by beef animals, and that dairy animals in particular were frequently moved between pastures [54]. This suggests that even distal parcels on highly fragmented land may be utilised at least some of the time. Furthermore, as we had only GIS shapefiles of land parcel boundaries, and no information on whether the boundary was a bio-secure barrier (e.g. mature hedgerow), the level of contact between neighbouring farms is likely to be biased by overestimation. However, previous studies have found that between $67 \%-79 \%$ of farm boundaries permit nose-to-nose contact [12, 32], so whilst we acknowledge the measurement error, we posit that the impact on the conclusions are minimal.

A limitation on determining the true extent of farms is introduced as a consequence of how the Basic Payment Scheme (BPS) system is administered. Either the land owner or tenant can claim for BPS and it is not possible to discern who is claiming for what parcels, and thus it is more challenging to be certain what land is being used and by whom. However, the BPS claimant is more often the farmer leasing and farming the land, and 
not the owner. This means that in practice, our estimations of farm area should largely reflect who is actively using the land parcels.

One constraint to our conclusions surrounds the lateral testing process. Because bTB disclosure triggers an intensive epidemiological investigation into the herds and parcels surrounding a breakdown herd, surveillance efforts are not homogenous. Thus, there may be increased likelihood of disease being identified proximal to case herds, based on this factor alone. Whilst we hypothesise that high odds ratio associated with the presence of neighbours reflects the contiguous spread of disease; it could be debated that we are observing a consequence of enhanced surveillance and increased intensity of testing. We, however, argue against this interpretation; due to the annual testing schedule, bTB would be eventually detected in the herds surrounding control herds if it were present.

\subsection{Future work}

The issue of fragmented farms poses an epidemiological risk for a wide range of pathogens in addition to bTB (e.g. BVD), however highly fragmented farms may also introduce logistical complexities into production, thereby decreasing the technical efficiency of dairy farms by [55]. Future work should therefore consider whether farm fragmentation in NI is a barrier to increased agricultural output. Furthermore, further studies to assess spatial patterns of conacre use in NI should be carried out, along with a quantification of the contribution of conacre to farm fragmentation. Tackling the bTB epidemic will require a more in-depth understanding of this highly localised phenomena, including the economic drivers and biosecurity implications of conacre land.

There may also be various landscapes effects influencing transmission risk on-farm [39, 56], if, for example, spillback risk may vary with land use heterogeneity.

\section{Materials and Methods}

We conducted a retrospective matched case-control observational study at the herd level to quantify the strength of association between the risk of bTB breakdown, and key metrics of farm fragmentation, fragment dispersal, and contact with contiguous farms. The study period ran between 01/01/2015 - 31/12/2017 inclusive.

\subsection{Study region and study population}

Northern Ireland (approximately 13,500km2) is situated in the northeast of the island of Ireland, with a national herd of 1.6 million cattle distributed throughout approximately 20,000 farms. Approximately $13 \%$ of these holdings are dairy farms $(n=2,600)$ and $70 \%$ are beef $(n=14,000)$, with a number of other herd types making up the remainder (e.g. breeding bulls) [35]. Cattle controls for $M$. bovis require that all bovines in NI over 42 days of age undergo annual testing using the Single Intradermal Comparative Cervical Tuberculin (SICCT) test, whereby SICCT-positive animals are removed from the herd and culled. Surveillance also involves the routine inspection of animal carcasses for lesions consistent with tuberculosis (LRS); suspect lesions are confirmed either way via histology or bacteriological investigations. We followed the Department for Agriculture, Environment and Rural Affairs (DAERA) policy on bTB breakdown confirmation at the time [36], which requires meeting one of the following criteria; 1) a single animal with positive result to two of the following confirmatory tests (the SICCT test, abattoir inspection for lesions, histology, and bacteriology), or 2) the presence of five or more LRS animals during the course of a breakdown.

\subsection{Exposure variables}

\subsubsection{Herd variables}

Data on individual cattle, cattle herd demographics, and bTB tests and breakdowns were provided by the DAERA Animal and Public Health Information System (APHIS) 
[37]. APHIS variables for individual cattle herds were the median herd size for the calendar year, the year of bTB testing, the herd bTB status (positive herds had at least one confirmed breakdown in the calendar year) the Divisional Veterinary Office wherein the farm homestead was recorded (DVO), and the herd type (Table 1).

\subsubsection{Spatial variables}

Land parcel spatial boundaries for every land parcel associated with each cattle farm were made available from the DAERA Land Parcel Identification System, and used to derive model variables. The sum of all land parcels was used to derive the total farm area (ha). We also calculated the number of "fragments" associated with each cattle business, with a fragment defined as a spatially distinct functional unit of land parcels. This was more appropriate than using the total number land parcels, as all the land parcels within a fragment are epidemiologically linked, as farmers permit cattle to move between adjoining fields. Fragments were defined in a previous study [27], but briefly all parcels belonging to a single farm within $5 \mathrm{~m}$ of each other were aggregated together into a single unit. This distance is width of a narrow access road in NI, and therefore represents a meaningful bio-secure boundary.

Fragment dispersal was defined using the median distance between fragments, calculated by measuring the Euclidian distance in $\mathrm{km}$ between fragment centroids. Contact with neighbouring farms was the length of shared perimeter between a cattle farm and its first order spatial neighbours (Figure 1). As it is known that some land parcels may be used for non-cattle activities, this was mitigated where possible by using Land Cover Map (LCM) data to determine which land parcels were potentially suitable for cattle farming (LCM classification 4 "improved grassland" and LCM classification 5 "neutral grassland"). This excludes land parcels classified as e.g. arable, woodland, bog, mountain or coast. Land Cover Maps for Northern Ireland for 2015 were purchased from the Centre for Ecology and Hydrology (https://www.ceh.ac.uk/) at $25 \mathrm{~m}^{2}$ resolution [37]. Farm fragmentation, fragment dispersal and contact with first order spatial neighbours were recorded as continuous variables and recoded as categorical exposures; both continuous and categorical variables were considered in this analysis. Farm fragmentation was split into five categories, and fragment dispersal and fragment area were each split into four quartiles (Table 1). Lastly, for each herd, we derived the number contiguous herds in which a bTB breakdown was confirmed in the twelve months prior to breakdown. 


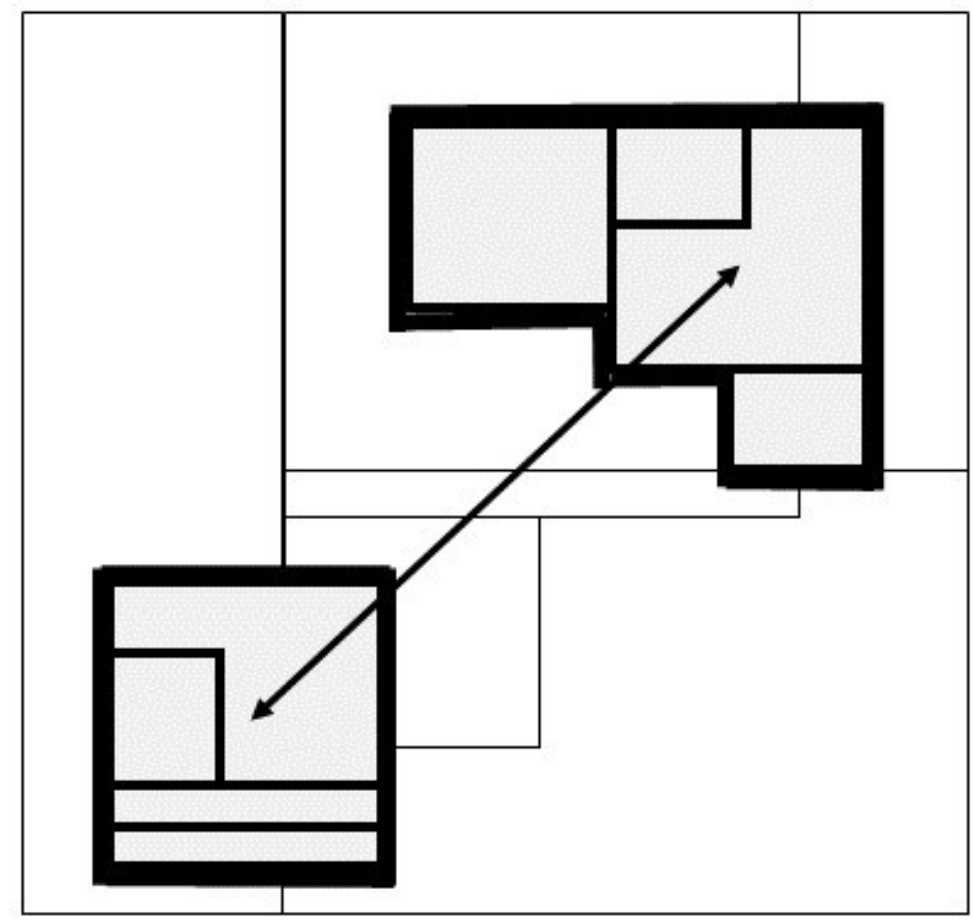

Figure 2. A schematic of definitions used in this manuscript. In this example, the index farm is shown in light grey, surrounded by land parcels belonging to first-order spatial neighbours in white. The index farm has eight land-parcels distributed across two fragments. The distance between the fragment centroids (in $\mathrm{km}$ ) is shown by the double headed arrow. The contact with neighbouring farms (in $\mathrm{km}$ ) is the perimeter of the fragments, coloured with the thickest black line.

Whilst similar, each of farm fragmentation, fragment dispersal and contact with neighbouring farms represent different exposures. We argue that number of first order spatial neighbours with bTB is the most specific measure of direct risk from contiguous herds, however we included the farm fragmentation variable as a separate measure as highly fragmented farms may be materially different from non-fragmented farms via management approaches related to grazing regime e.g. rotational grazing across multiple parcels. Calculating the length of shared boundary with neighbouring cattle farms is a more precise measure of contact with neighbouring herds, given that this metric was derived from boundaries where both internal and external parcels were potentially suitable for cattle farming. We also included fragment dispersal, as unrecorded, intra-herd cattle movements could lead to animals being moved from lower incidence areas to higher incidence areas, thereby increasing the risk of breakdown. In addition to this, widely dispersed land parcels may capture more landscape heterogeneity on-farm, with potentially better habitat provision for potential reservoir species via availability of rough pasture and woodland [38].

\subsection{Participating herds}

Case herds were those with at least one confirmed bTB breakdown that started and ended during the dates of the study. For herds that had more than one confirmed breakdown, we included only the earliest breakdown (although $76.7 \%$ of herds experienced only a single breakdown). Eligible control herds were herds that remained bTB free throughout the study period. We matched on production type (as it was previously found that dairy herds tend to be associated with higher levels of farm fragmentation, and different production types may experience differential risk of bTB breakdown); herd size (as larger herds may reside on more fragmented land, are known to be at 
higher risk of bTB); and Divisional Veterinary Office (DVO), a local spatial variable associated with bTB administration (this variable is known to capture spatial variation in bTB risk and wildlife density), and year of bTB test. Herd size was the only non-categorical matching variable, and here we matched on herd size $+/-10$ head of cattle. Cases and controls were matches in a 1:1 ratio on potential confounding variables; we use a higher ratio of controls to cases as it was not possible to match on all criteria.

\subsection{Analysis}

Descriptive statistics for each explanatory variable (counts and percentages for categorical variables; median and lower and upper Inter-Quartile Range (IQR) for continuous variables) were generated for case and control herds. Locally estimated scatterplot smoothing (LOESS) plots and boxplots were created for exploratory analysis. Correlations between explanatory variables were assessed using Spearman' Rank Correlation Coefficient. Univariable and multivariable parameter estimates were derived using conditional GLMs via the clogit function in the survival package [39]. Pair IDs were used to control the matching. Analyses were carried out using both continuous and categorical explanatory variables. The Odds Ratios (OR's) were reported, along with the 95\% Lower and Upper Confidence Intervals (95\% CI). The null hypothesis was that there is no association between the odds of bTB breakdown and each of the candidate exposures, after controlling for herd size, herd type, DVO and year of breakdown. Tests for linear trend (i.e. dose -response relationships) between bTB risk and categorical exposures were carried out by fitting models wherein the categories are treated as ordered factors as opposed to nominal categories. These simpler ordinal models were compared with the more complex categorical models using Likelihood Ratio Tests (LRTs). Here, the null hypothesis was that the simpler, ordinal model sufficiently fits the data. Furthermore, we hypothesise that that the number of bTB positive neighbours is likely to confound the relationship between bTB breakdown risk and each of farm area, farm fragmentation, fragment dispersal and contact with neighbouring farms. We considered confounding effects to be present where changes of $10 \%$ or more were observed in parameter estimates, after the infectious neighbours variable was added to the model [40]. We were particularly interested in any remaining association between the odds of bTB breakdown and each of farm area, farm fragmentation, fragment dispersal and contact with neighbouring farms, once the number of bTB positive neighbours was taken into account. This would suggest additional risk associated with these attributes beyond the presence of over the fence contact with infectious herds; here, we report the adjusted Odds Ratio (aOR) and 95\%CI's. The full analytical procedure is presented in the R Markdown output (Supplementary Material 1). Figures were generated using ggplot2.

\section{Conclusions}

Northern Ireland's agricultural system means that many farms are very fragmented, resulting in herds with high levels of direct contact with neighbouring farms, and sometimes considerable distances between fragments. We were ultimately interested in whether fragmentation was associated with odds of bTB breakdown, and whilst we found that farm area, farm fragmentation, fragment dispersal and contact with neighbouring farms were indeed associated with increased odds of breakdown, these factors were also confounded with the number of bTB positive neighbours. We found that highly fragmented farms were around twice as likely to have at least one bTB positive neighbour compared to non-fragmented farms, and that every bTB positive neighbour increased the odds of bTB breakdown by $40 \%-50 \%$. After accounting for the number of bTB positive neighbours in models of how bTB breakdown risk is associated with farm fragmentation metrics, we find no compelling evidence of a relationship between odds of bTB breakdown and fragmentation. This signifies that these farm fragmentation metrics are probably capturing the effects of "over the fence" cattle-to-cattle transmission. Although it is 
possible that similar observations arise if herds in the area were exposed to a shared infection source such as infected wildlife, our geographical matching criteria makes this less likely. Tackling local spread will require a deeper understanding of the patterns, drivers and characteristics of NI's conacre rental system, which is a key factor influencing land fragmentation.

Supplementary Materials: The following are available online at www.mdpi.com/xxx/s1.

Author Contributions: Conceptualization, AWB; methodology, GM; software, JG; formal analysis, GM; resources, JMcG, WMcM, RK; data curation, JMcG, WMcM, RK; writing-original draft prepara-tion, GM and AWB; writing - review and editing, all co-authors.; visualization, GM; project administration, GM; funding acquisition, AWB. All authors have read and agreed to the published version of the manuscript.

Funding: This work was supported by the Department of Agriculture, Environment and Rural Affairs (DAERA), and was fully funded under grant 18/3/02 (48258) - FaRTHEr: Fragmentation As a Risk factor for TB in cattle Herds: impacts on Eradication.

Institutional Review Board Statement: Not applicable; all disease data were collected as part of routine disease surveillance.

Informed Consent Statement: Not applicable. 
Data Availability Statement: An anonymisec version of the dataset used in this analysis is available upon request from the corrosponding author.

\begin{abstract}
Acknowledgments: The authors also to extend thanks and acknowledgement to all of the AFBI staff involved in bovine tuberculosis histology, pathology, bacteriology, molecular microbiology, case confirmation and MLVA typing, along with the Epidemiology, Molecular biology and Immunology cluster (https://twitter.com/AFBI_EMbI) for technical support and discussion. We are also thankful to the DAERA Veterinary Service and abattoir staff who deliver the NI bTB eradication programme, those staff who maintain and manage the DAERA APHIS database, and the staff who maintain and manage the LPIS dataset and administer the Area Based Scheme.
\end{abstract}

Conflicts of Interest: The authors declare no conflict of interest. Co-authors JMcG, WMcM and RK work for the funding organization, but did not fund this project directly. These individuals have roles in providing data resources, data curation, writing and review and editing

\title{
References
}

1. 1. Humblet, M.-F., M.L. Boschiroli, and C. Saegerman, Classification of worldwide bovine tuberculosis risk factors in cattle: 1. Humblet M-F, Boschiroli ML, Saegerman C. Classification of worldwide bovine tuberculosis risk factors in cattle: a stratified approach. Veterinary Research. 2009;40(5):50.

2. 2. More SJ. Can bovine TB be eradicated from the Republic of Ireland? Could this be achieved by 2030? Irish Veterinary Journal. 2019;72(1):3.

3. 3. Robinson PA. A history of bovine tuberculosis eradication policy in Northern Ireland. Epidemiology and Infection. 2015;143(15):3182-95.

4. 4. Woods A. A historical synopsis of farm animal disease and public policy in twentieth century Britain. Philosophical Transactions of the Royal Society B: Biological Sciences. 2011;366(1573):1943-54.

5. 5. Health EPoA, Welfare. Scientific Opinion on the use of a gamma interferon test for the diagnosis of bovine tuberculosis: bovine TB Test. EFSA Journal. 2012;10(12):2975.

6. 6. Lahuerta-Marin A, Milne MG, McNair J, Skuce RA, McBride SH, Menzies FD, et al. Bayesian latent class estimation of sensitivity and specificity parameters of diagnostic tests for bovine tuberculosis in chronically infected herds in Northern Ireland. Veterinary journal (London, England : 1997). 2018;238:15-21.

7. 7. Clegg TA, Doyle M, Ryan E, More SJ, Gormley E. Characteristics of Mycobacterium bovis infected herds tested with the interferon-gamma assay. Preventive veterinary medicine. 2019.

8. 8. Pascual-Linaza AV, Gordon AW, Stringer LA, Menzies FD. Efficiency of slaughterhouse surveillance for the detection of bovine tuberculosis in cattle in Northern Ireland. Epidemiology and Infection. 2017;145(05):995-1005.

9. 9. McKinley TJ, Lipschutz-Powell D, Mitchell AP, Wood JLN, Conlan AJK. Risk factors and variations in detection of new bovine tuberculosis breakdowns via slaughterhouse surveillance in Great Britain. PLOS ONE. 2018;13(6):e0198760.

10. 10.Allen AR, Skuce RA, Byrne AW. Bovine Tuberculosis in Britain and Ireland - A Perfect Storm? the Confluence of Potential Ecological and Epidemiological Impediments to Controlling a Chronic Infectious Disease. Frontiers in Veterinary Science. 2018;5(109).

11. 11.Ramírez-Villaescusa AM, Medley GF, Mason S, Green LE. Risk factors for herd breakdown with bovine tuberculosis in 148 cattle herds in the south west of England. Preventive veterinary medicine. 2010;95(3-4):224-30.

12. 12. O'Hagan MJH, Matthews DI, Laird C, McDowell SWJ. Herd-level risk factors for bovine tuberculosis and adoption of related biosecurity measures in Northern Ireland: A case-control study. The Veterinary Journal. 2016;213:26-32.

13. 13. Gopal R, Goodchild A, Hewinson G, Domenech RdlR, Clifton-Hadley R. Introduction of bovine tuberculosis to northeast England by bought-in cattle. Veterinary Record. 2006;159(9):265-71.

14. 14. Green DM, Kiss IZ, Mitchell AP, Kao RR. Estimates for local and movement-based transmission of bovine tuberculosis in British cattle. Proceedings of the Royal Society of London B: Biological Sciences. 2008;275(1638):1001-5.

15. 15.Vial F, Johnston WT, Donnelly CA. Local Cattle and Badger Populations Affect the Risk of Confirmed Tuberculosis in British Cattle Herds. PLOS ONE. 2011;6(3):e18058.

16. 16.Johnston WT, Vial F, Gettinby G, Bourne FJ, Clifton-Hadley RS, Cox DR, et al. Herd-level risk factors of bovine tuberculosis in England and Wales after the 2001 foot-and-mouth disease epidemic. International Journal of Infectious Diseases. 2011;15(12):e833-e40.

17. 17. Milne G, Allen A, Graham J, Lahuerta-Marin A, McCormick C, Presho E, et al. Bovine tuberculosis breakdown duration in cattle herds: an investigation of herd, host, pathogen and wildlife risk factors. PeerJ. 2020;8:e8319.

18. 18. Byrne AW, Kenny K, Fogarty U, O’Keeffe JJ, More SJ, McGrath G, et al. Spatial and temporal analyses of metrics of tuberculosis infection in badgers (Meles meles) from the Republic of Ireland: Trends in apparent prevalence. Preventive veterinary medicine. 2015;122(3):345-54.

19. 19.Wright DM, Reid N, Ian Montgomery W, Allen AR, Skuce RA, Kao RR. Herd-level bovine tuberculosis risk factors: assessing the role of low-level badger population disturbance. Scientific Reports. 2015;5:13062. 
20. 20.Campbell EL, Byrne AW, Menzies FD, McBride KR, McCormick CM, Scantlebury M, et al. Interspecific visitation of cattle and badgers to fomites: A transmission risk for bovine tuberculosis? Ecology and Evolution. 2019.

21. 21. Woodroffe R, Donnelly CA, Cox DR, Gilks P, Jenkins HE, Johnston WT, et al. Bovine tuberculosis in cattle and badgers in localized culling areas. J Wildl Dis. 2009;45(1):128-43.

22. 22. White PW, Martin SW, De Jong MCM, O'Keeffe JJ, More SJ, Frankena K. The importance of 'neighbourhood' in the persistence of bovine tuberculosis in Irish cattle herds. Preventive veterinary medicine. 2013;110(3-4):346-55.

23. 23. Griffin JM, Martin SW, Thorburn MA, Eves JA, Hammond RF. A case-control study on the association of selected risk factors with the occurrence of bovine tuberculosis in the Republic of Ireland. Preventive veterinary medicine. 1996;27(12):75-87.

24. 24. Young JS, Gormley E, Wellington EMH. Molecular Detection of Mycobacterium bovis and Mycobacterium bovis BCG (Pasteur) in Soil. Applied and Environmental Microbiology. 2005;71(4):1946-52.

25. 25.NIAO. The control of Bovine Tuberculosis in Northern Ireland. Belfast: Northern Ireland Audit Office; 2018 27/11/2018.

26. 26.EC. Eradication programme for Bovine Tuberculosis - United Kingdom. Commission européenne, B-1049 Bruxelles; 2013. Contract No.: SANCO/10341/2013.

27. 27. Byrne AW, Barrett D, Breslin P, Madden JM, Keeffe J, Ryan E. Bovine Tuberculosis (Mycobacterium bovis) outbreak duration in cattle herds in Ireland: A retrospective observational study. Pathogens. 2020;9(10).

28. 28. Broughan JM, Maye D, Carmody P, Brunton LA, Ashton A, Wint W, et al. Farm characteristics and farmer perceptions associated with bovine tuberculosis incidents in areas of emerging endemic spread. Preventive veterinary medicine. 2016;129:88-98.

29. 29.Independent Scientific Group on Cattle TB. Bovine TB: The Scientific Evidence. A Science Base for a Sustainable Policy to Control TB in Cattle. 2007.

30. 30.Brennan ML, Kemp R, Christley RM. Direct and indirect contacts between cattle farms in north-west England. Preventive veterinary medicine. 2008;84(3-4):242-60.

31. 31.Denny GO, Wilesmith JW. Bovine tuberculosis in Northern Ireland: case-control study of herd risk factors. The Veterinary Record. 1999;144:310.

32. 32. Campbell EL, Menzies FD, Byrne AW, Porter S, McCormick CM, McBride KR, et al. Grazing cattle exposure to neighbouring herds and badgers in relation to bovine tuberculosis risk. Research in Veterinary Science. 2020.

33. 33. Milne MG, Graham J, Allen A, McCormick C, Presho E, Skuce R, et al. Variation in Mycobacterium bovis genetic richness suggests that inwards cattle movements are a more important source of infection in beef herds than in dairy herds. BMC Microbiology. 2019;19(1):154.

34. 34.DAERA. Final Results of the June Agricultural Census 2019. Belfast: Statistics and Analytical Services Branch, Policy, Economics and Statistics Division 2020. Available from: https://www.daera-ni.gov.uk/sites/default/files/publications/daera/Final\%20Results\%20of\%20the\%20June\%202019\%20Agricultural\%20Census.pdf.

35. 35.DAERA. Bovine Tuberculosis (TB) testing I Department of Agriculture, Environment and Rural Affairs. 2017.

36. 36. Houston R. A computerised database system for bovine traceability. Revue Scientifique et Technique-Office International des Epizooties. 2001;20(2):652.

37. 37. Rowland CS, Morton RD, Carrasco L, McShane G, O'Neil AW, Wood CM. Land Cover Map 2015 (vector, N. Ireland). NERC Environmental Information Data Centre; 2017.

38. 38.Winkler B, Mathews F. Environmental risk factors associated with bovine tuberculosis among cattle in high-risk areas. Biology Letters. 2015;11(11):20150536.

39. 39. Therneau TM. A Package for Survival Analysis in R. R package version 3.2-13 ed2021. p. R package version 3.2-13.

40. 40.Dohoo I, Martin W, Stryhn H. Veterinary Epidemiologic Research. 2nd edition ed. Charlottetown, CA: VER Inc; 2009 2009. 865 p.

41. 41.Skuce RA, Mallon TR, McCormick CM, McBride SH, Clarke G, Thompson A, et al. Mycobacterium bovis genotypes in Northern Ireland: herd-level surveillance (2003 to 2008). Veterinary Record. 2010;167(18):684-9.

42. 42.Biek R, O'Hare A, Wright D, Mallon T, McCormick C, Orton RJ, et al. Whole Genome Sequencing Reveals Local Transmission Patterns of Mycobacterium bovis in Sympatric Cattle and Badger Populations. PLOS Pathogens. 2012;8(11):e1003008.

43. 43. Milne G, Allen A, Graham J, Kirke R, McCormick C, Presho E, et al. Mycobacterium bovis population structure in cattle and local badgers: Co-localisation and variation by farm type. Pathogens. 2020;9(7):592.

44. 44. Trewby $\mathrm{H}$. The genetic and spatial epidemiology of bovine tuberculosis in the UK : from molecular typing to bacterial whole genome sequencing University of Glasgow; 2016.

45. 45.Johnston WT, Gettinby G, Cox DR, Donnelly CA, Bourne J, Clifton-Hadley R, et al. Herd-level risk factors associated with tuberculosis breakdowns among cattle herds in England before the 2001 foot-and-mouth disease epidemic. Biology Letters. 2005;1(1):53-6.

46. 46. Mill AC, Rushton SP, Shirley MDF, Murray AWA, Smith GC, Delahay RJ, et al. Farm-scale risk factors for bovine tuberculosis incidence in cattle herds during the Randomized Badger Culling Trial. Epidemiology and Infection. 2012;140(2):21930 .

47. 47.Milne MG, Byrne AW, Campbell E, Graham J, McGrath J, Kirke R, et al. Quantifying Land Fragmentation Metrics for Cattle Enterprises in Northern Ireland. Preprints. 2021;2021100149. 
48. 48. Enright J, Kao RR. A descriptive analysis of the growth of unrecorded interactions amongst cattle-raising premises in Scotland and their implications for disease spread. BMC Veterinary Research. 2016;12(1):37.

49. 49. Orton RJ, Bessell PR, Birch CPD, O'Hare A, Kao RR. Risk of Foot-and-Mouth Disease Spread Due to Sole Occupancy Authorities and Linked Cattle Holdings. PLOS ONE. 2012;7(4):e35089.

50. 50.Doyle LP, Courcier EA, Gordon AW, O'Hagan MJH, Johnston P, McAleese E, et al. Northern Ireland farm-level management factors for prolonged bovine tuberculosis herd breakdowns. Epidemiology and infection. 2020;148:e234-e.

51. 51.Brown E, Marshall AH, Mitchell HJ, Byrne AW. Cattle movements in Northern Ireland form a robust network: implications for disease management. Preventive veterinary medicine. 2019;170:104740.

52. 52. Adenuga AH, Jack C, McCarry R. The Case for Long-Term Land Leasing: A Review of the Empirical Literature. Land. 2021;10(3):238.

53. 53. Cathal G, Anne K, Cathal OD. The effect of farmer attitudes on openness to land transactions: evidence for Ireland. Biobased and Applied Economics. 2021;10(2).

54. 54.Campbell EL, Byrne AW, Menzies FD, Milne G, McBride KR, McCormick CM, et al. Quantifying intraherd cattle movement metrics: Implications for disease transmission risk. Preventive veterinary medicine. 2020:105203.

55. 55.Bradfield T, Butler R, Dillon E, Hennessy T, Kilgarriff P. The Effect of Land Fragmentation on the Technical Inefficiency of Dairy Farms. Journal of Agricultural Economics. 2020;72.

56. 56. White PCL, Brown JA, Harris S. Badgers Meles meles, cattle and bovine tuberculosis Mycobacterium bovis : a hypothesis to explain the influence of habitat on the risk of disease transmission in southwest England. Proceedings of the Royal Society of London Series B: Biological Sciences. 1993;253(1338):277-84. 